\title{
Top quark pair charge asymmetry and spin correlation measurements at the LHC
}

\section{Thorsten Chwalek*}

On behalf of the ATLAS and CMS Collaborations.

Karlsruhe Institute of Technology (DE)

E-mail: chwalek@cern.ch

\begin{abstract}
We give an overview over charge asymmetry and spin correlation measurements in top quark pair production. The presented analyses use data collected with the ATLAS and CMS detectors at a center-of-mass energy of $8 \mathrm{TeV}$. Events with either dileptonic or semileptonic top quark pair decays are analyzed. All results are comparable with the predictions by the standard model.
\end{abstract}

Fourth Annual Large Hadron Collider Physics

13-18 June 2016

Lund, Sweden

${ }^{*}$ Speaker. 


\section{Introduction}

Top quarks are the heaviest elementary particles known to date. Consequently they decay almost immediately after production without taking part in hadronization processes. As a result the information on their initial direction and spin orientation is preserved in the kinematics of their decay products. Either by fully reconstructing the kinematics of the top quark-antiquark pair (tt) or by looking at angular distributions of the leptons from semileptonic top quark decays several properties of the heaviest quark can be analyzed. In the following, measurements of the $\mathrm{t} \overline{\mathrm{t}}$ charge asymmetry and the spin correlation of top quark and antiquark are briefly summarized. The described analyses use proton proton collision data at a center-of-mass energy of $\sqrt{s}=8 \mathrm{TeV}$, collected by the ATLAS [1] or CMS [2] detector at the CERN LHC.

\section{Spin correlation}

The theory of quantum chromodynamics predicts that in the production of pairs of heavy quarks their spins are correlated. As top quarks decay faster than the timescale at which decorrelating effects typically happen, this spin correlation is conserved and propagated to the top quark decay products and can be analyzed by looking at angular distributions of these decay products. Depending on the $t \bar{t}$ production mode and the choice of a spin quantization axis one defines a spin correlation strength $A$ as the asymmetry between the number of events in which the spins of the t⿱t pair are aligned or anti-aligned. This can also be expressed by the fraction of $t \bar{t}$ events with standard model (SM) spin correlation with respect to the total number of selected $t \bar{t}$ events, including potential contributions from $\mathrm{t} \overline{\mathrm{t}}$ events with uncorrelated spins, $f=N_{\text {cor }}^{\mathrm{t}} /\left(N_{\text {cor }}^{\mathrm{t} \overline{\mathrm{t}}}+N_{\mathrm{uncor}}^{\mathrm{t} \overline{\mathrm{t}}}\right)$.

\subsection{Measurement of spin correlation in dileptonic $t \bar{t}$ events at ATLAS}

The ATLAS Collaboration uses a dataset corresponding to an integrated luminosity of $20.3 \mathrm{fb}^{-1}$ to measure the $\overline{\mathrm{t}} \mathrm{t}$ spin correlation in dilepton events (ee, $\mu \mu$, and $\mathrm{e} \mu$ channels) [3]. The azimuthal angle between the two charged leptons, $\Delta \phi$, is used as sensitive variable. As this variable is defined in the laboratory frame, no top quark reconstruction is needed. The observed $\Delta \phi$ distribution is fitted using two different templates for t⿱t production, one with SM spin correlation included and one without spin correlation. The background normalization is fixed in the fit. Figure 1 (upper left) shows the $\Delta \phi$ distribution observed in data, as well as the theory predictions for t⿱t production with and without spin correlations and the fit result. The measured value of the coefficient $f$, measuring the degree of spin correlation relative to the SM prediction, is $f=1.20 \pm 0.05$ (stat) \pm 0.13 (syst). This result can be transformed into a value for the spin correlation strength $A_{\text {helicity }}$, defined in the helicity base, by multiplying the SM predicted value of $A_{\text {helicity }}^{\mathrm{SM}}=0.318 \pm 0.005$ with the measured $f$ value, yielding $A_{\text {helicity }}=0.38 \pm 0.04$ (stat $\oplus$ syst).

A specific model that predicts zero spin correlation is the production of supersymmetric top squarks, where the top squarks decay via $\tilde{\mathfrak{t}}_{1} \rightarrow \mathrm{t} \tilde{\chi}_{1}^{0}$ into top quark and neutralino. The $\Delta \phi$ distribution is also used to extract limits on the cross section of such processes, as a function of the mass of the supersymmetric partner of the top quark. Figure 1(upper right) shows the obtained limits. Assuming a $100 \%$ branching ratio for $\tilde{\mathrm{t}}_{1} \rightarrow \mathrm{t} \tilde{\chi}_{1}^{0}$ and a neutralino mass of $1 \mathrm{GeV}$, top squark masses between the top quark mass and $191 \mathrm{GeV}$ are excluded, where the expected limit is $178 \mathrm{GeV}$. 

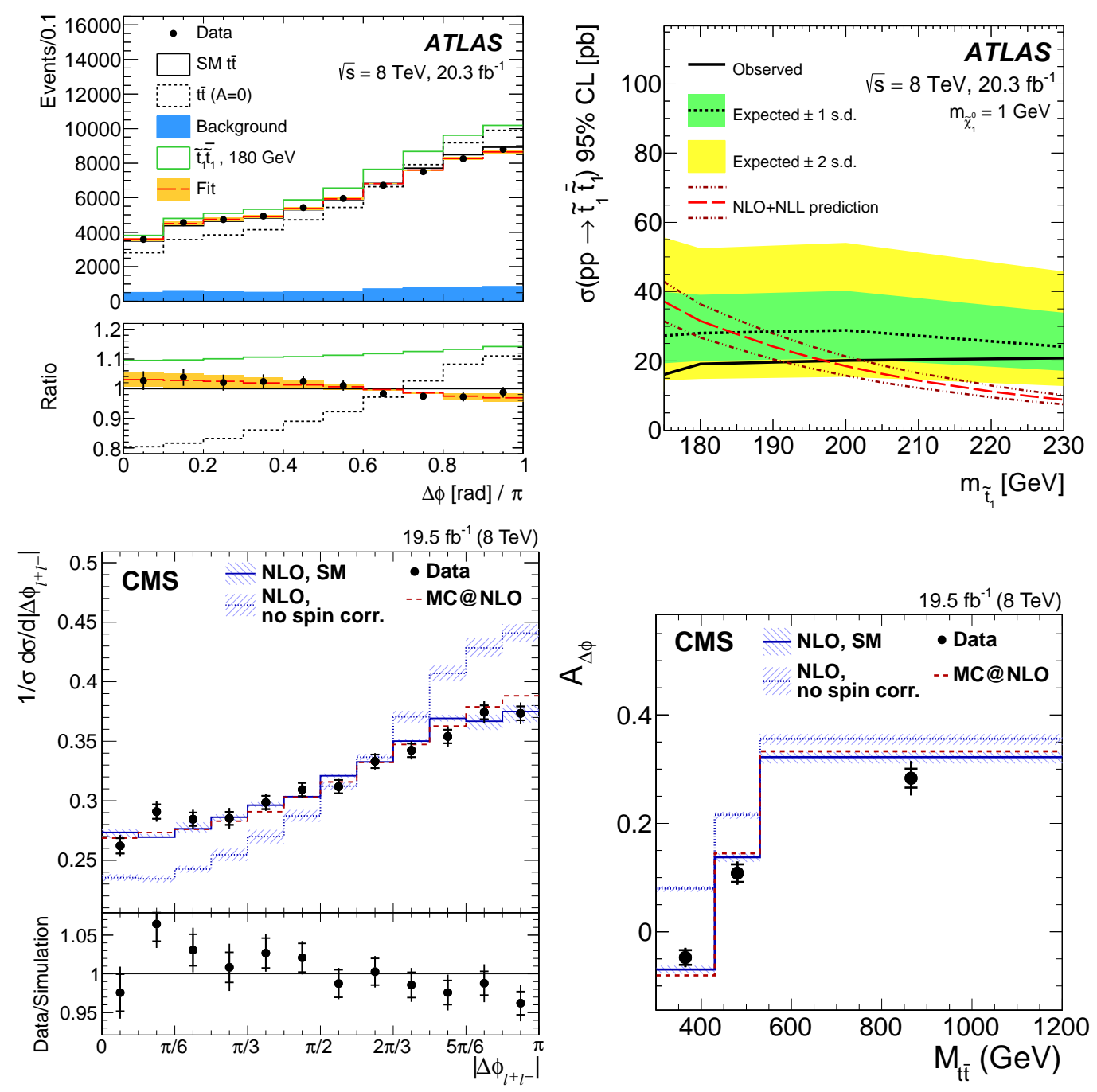

Figure 1: Spin correlation in dilepton events, upper row ATLAS [3], bottom row CMS [5]. Upper left: Reconstructed $\Delta \phi$ distribution for the sum of the three dilepton channels in data in comparison to the prediction for the SM and to the prediction for no spin correlation. The green histogram shows the prediction for $\tilde{\mathfrak{t}}_{1} \overline{\tilde{t}}_{1}$ production $\left(m_{\tilde{\mathfrak{t}}_{1}}=180 \mathrm{GeV}\right.$ and $\left.m_{\tilde{\chi}_{1}^{0}}=1 \mathrm{GeV}\right)$. The lower panel shows the distributions divided by the SM tit prediction plus background. Upper right: Expected and observed limits at 95\% CL on the top squark pair-production cross section as a function of $m_{\tilde{t}_{1}}$, for top squark decays $\tilde{\mathrm{t}}_{1} \rightarrow \mathrm{t} \tilde{\chi}_{1}^{0}$ with $m_{\tilde{\chi}_{1}^{0}}=1 \mathrm{GeV}$. Bottom left: Normalized differential cross section as a function of $\left|\Delta \phi_{\ell^{+} \ell^{-}}\right|$in data compared to theory predictions for SM and no spin correlation. The lower panel shows the ratio of data to the MC@NLO [4] simulation. Bottom right: Dependence of the asymmetry variable $\mathrm{A}_{\Delta \phi}$ on the invariant mass of the $\bar{t} \overline{\mathrm{t}}$ system compared to theory predictions for SM and no spin correlation.

\subsection{Measurement of spin correlation in dileptonic tit events at CMS}

The same channels are analyzed by the CMS Collaboration using $19.5 \mathrm{fb}^{-1}$ of data [5]. In this analysis, several variables are examined that are sensitive to the tit spin correlation: $\Delta \phi$ between the two charged leptons in the laboratory frame; the opening angle $\varphi$ between the two leptons, 
measured in the rest frames of their respective parent top (anti)quark; the product of the cosines of the helicity angles of the two leptons, $\cos \theta_{\ell^{+}}^{*} \cos \theta_{\ell^{-}}^{*}$. In contrast to $\Delta \phi$, the latter two variables require the full reconstruction of the t⿱t system. The reconstructed distributions are unfolded to the parton level to allow for a direct comparison with theoretical predictions. From the unfolded and normalized differential cross sections as a function of the described variables the asymmetries are determined. This is done inclusively and double-differentially as functions of variables characterizing the $\bar{t} \bar{t}$ system: the invariant mass $\left(m_{\overline{\mathrm{t}}}\right)$, the rapidity $\left(y_{\bar{t} \mathrm{t}}\right)$, and the transverse momentum $\left(p_{\mathrm{T}}^{\mathrm{t}}\right)$ of the t⿱t system. Figure 1 (bottom left) shows the unfolded $|\Delta \phi|$ distribution in data together with theoretical predictions for $\bar{t}$ production with and without spin correlation as an example for the inclusive measurements in the different sensitive variables. The measured asymmetries using this variable in three different regions of $m_{\mathrm{tt}}$ are shown in Fig. 1 (bottom right). All measured asym-

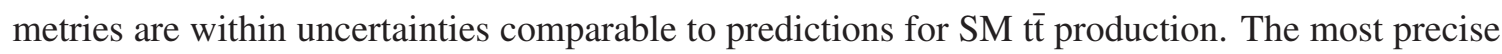
result comes from the projection in $|\Delta \phi|$ of the double-differential cross section as a function of $|\Delta \phi|$ and $m_{\mathrm{tt}}$ and is $A_{\Delta \phi}=0.095 \pm 0.006$ (stat) \pm 0.007 (syst), which is converted into $f=1.12_{-0.15}^{+0.12}$.

\subsection{Measurement of spin correlation in muon+jets tt events at CMS}

The CMS Collaboration analyzes $19.7 \mathrm{fb}^{-1}$ of data in the muon+jets channel to test the hypothesis that $t \bar{t}$ events are produced with spin correlation as predicted by the SM [6]. The analysis makes use of a matrix element method to calculate for each event the ratio between the likelihood under the SM hypothesis and the fully uncorrelated hypothesis, $\lambda_{\text {event }}=\mathrm{P}\left(\mathrm{H}_{\text {uncor }}\right) / \mathrm{P}\left(\mathrm{H}_{\text {cor }}\right)$. By fitting the $\lambda_{\text {event }}$ distribution with templates from simulated $\bar{t} \bar{t}$ samples, produced with and with-
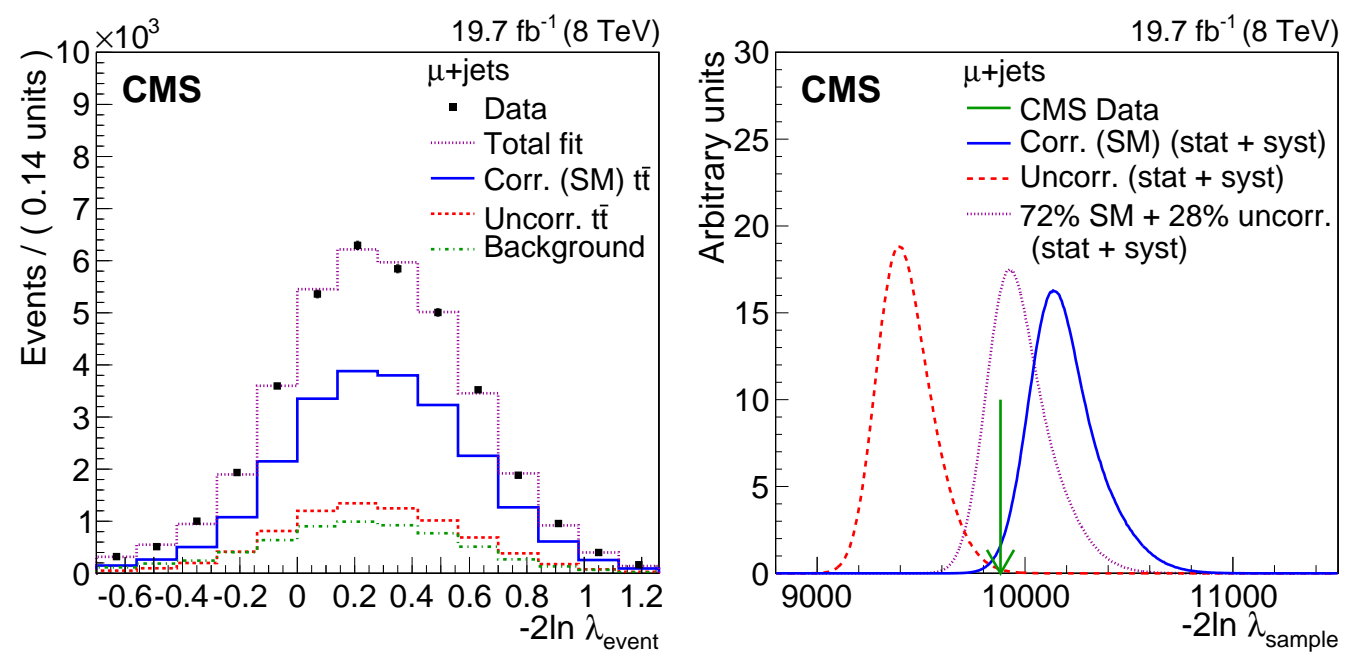

Figure 2: Spin correlation in muon+jets events [6]. Left: Result of the template fit to data. The solid curve represents the contribution of the SM $\bar{t} \bar{t}$ production, the dashed curve is the contribution of $\bar{t} \bar{t}$ production without spin correlation. Right: The $2 \ln \lambda_{\text {sample }}$ distribution in simulation, evaluated for the data set size. The samples in simulation contain signal and background mixed according to the theoretical cross sections, with

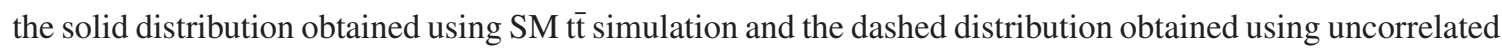
$\mathrm{t} \overline{\mathrm{t}}$ simulation, including systematic uncertainties. The arrow indicates the $2 \ln \lambda_{\text {sample }}$ observed in data. The dotted curve shows a mixture of $72 \% \mathrm{SM}$ t⿱t events and $28 \%$ uncorrelated $\overline{\mathrm{t}} \overline{\mathrm{t}}$ events. 
out spin correlations, the fraction $f$ of signal events with SM spin correlation is found to be $f=0.72 \pm 0.08(\mathrm{stat})_{-0.13}^{+0.15}$ (syst). Similar as in the ATLAS analysis, this result is converted into a value for $A_{\text {helicity }}$ by assuming a linear dependence between $A_{\text {helicity }}$ and $f, A_{\text {helicity }}=0.23 \pm$ 0.03 (stat) ${ }_{-0.04}^{+0.05}$ (syst). Figure 2 (left) shows the observed $-2 \ln \lambda_{\text {event }}$ distribution together with the single fit templates and the total fit result. Multiplying the event likelihoods for all events in the sample results in the so-called "sample likelihood", $\lambda_{\text {sample }}$. From repeated pseudo-experiments using a template morphing technique to take systematic uncertainties into account the $\lambda_{\text {sample }}$ distributions for the two hypotheses of correlated spins and uncorrelated spins, as shown in Fig. 2 (right) are obtained. The comparison with the value observed in data (green arrow in Fig. 2 (right)) yields a compatibility of the data with the hypothesis of uncorrelated spins within 2.9 standard deviations and a compatibility with the SM hypothesis within 2.2 standard deviations.

\section{Charge asymmetry}

Top quark pairs are produced via the strong interaction either from the fusion of two incoming gluons or from the annihilation of an incoming quark and an incoming antiquark. While the initial state with two gluons is charge symmetric, the quark-antiquark initial state is not. Interference effects between Born and box diagrams as well as between diagrams with initial and final state radiation connect the direction of the produced (anti)top quark to that of the incoming (anti)quark. In most cases the quark in the initial state is a valence quark inside one of the two colliding protons while the initial state anti-quark is from the quark-sea of the other proton. Valence quarks feature on average a harder transverse momentum spectrum than sea-quarks. This connection between initial (anti)quarks and produced top (anti)quarks manifests itself in an asymmetry in the angular distributions of top quarks and antiquarks. Top quarks are produced more centrally while top antiquarks have a slight preference for being produced in forward directions. Thus, the difference of the absolute values of the rapidity of top quark and antiquark in an event, $\Delta|y|$, is a sensitive variable to measure this effect. The asymmetry is then defined as the difference of the number of events with a positive value and the number of events with a negative value of this variable, divided by the sum of all events. SM calculations predict an asymmetry of about $1 \%$ at $\sqrt{s}=8 \mathrm{TeV}[7,8]$.

\subsection{Measurement of the $t \bar{t}$ charge asymmetry in lepton+jets events using a template method at CMS}

The most precise measurement of the inclusive $t \bar{t}$ charge asymmetry uses a template method to analyze $19.6 \mathrm{fb}^{-1}$ of data in the lepton+jets channel [9]. The analysis uses a transformation of $\Delta|y|, \Upsilon_{\mathrm{t}}=\tanh \Delta|y|$, as sensitive variable, as for this method a bounded variable is desirable. The rapidities of top quark and antiquark, needed for the calculation of this variable, are derived from fully reconstructing the $\bar{t} \bar{t}$ system by applying a kinematic fitting procedure. The template method expresses the differential cross section of $t \bar{t}$ production as a function of $\Upsilon_{\bar{t} t}$ as the sum of symmetric and antisymmetric components. The charge asymmetry is then determined from a fit to the reconstructed $\Upsilon_{\mathrm{tt}}$ distributions. The reconstructed and fitted antisymmetric component distributions for the e+jets and $\mu+$ jets channels can be found in Fig. 3. The measured charge asymmetry is $A_{C}=0.0033 \pm 0.0026$ (stat) \pm 0.0033 (syst), which is consistent with SM predictions. 

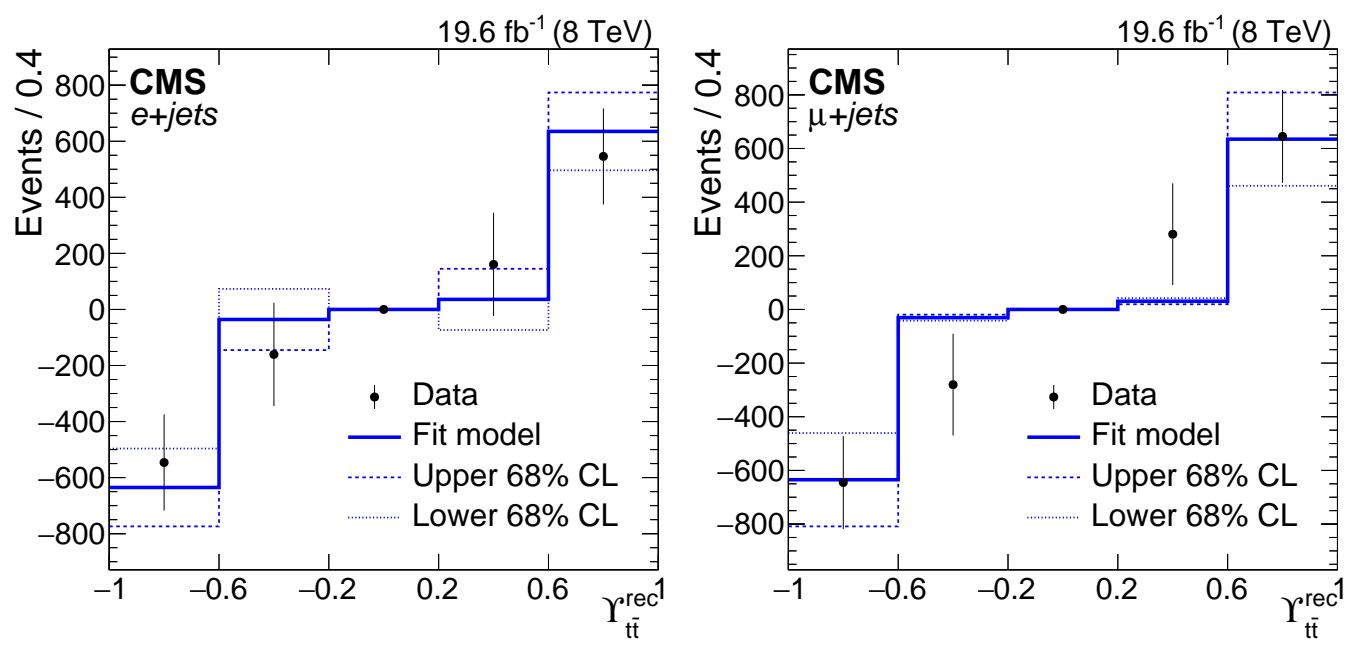

Figure 3: Charge asymmetry measurement using a template method [9]. The antisymmetric component of the $\Upsilon_{\mathrm{t}}^{\text {rec }}$ distributions in data and the model are shown for e+jets (left) and $\mu+$ jets (right), for the central value (solid), and for the upper (dashed) and lower (dotted) limits of the $68 \%$ statistical confidence intervals.

\subsection{Measurement of the t⿱t charge asymmetry in lepton+jets and dilepton events using an unfolding method at CMS}

In contrast to the previously described analysis another CMS analysis [10] corrects the reconstructed distribution of the sensitive variable $\Delta|y|$ back to parton level to compare it directly to theory predictions. This is done by applying an unfolding procedure (as implemented in the TUnfold package [11]) on the reconstructed $\Delta|y|$ distribution and calculating the charge asymmetry from the unfolded spectrum. At the cost of an increased statistical uncertainty, caused by the unfolding, this method enables not only inclusive but also differential measurements. The charge asymmetry is measured as function of kinematic variables characterizing the $\bar{t} \bar{t}$ system, the invariant mass $m_{\overline{\mathrm{tt}}}$, the absolute value of the rapidity $\left|y_{\mathrm{t}}\right|$, and the transverse momentum $p_{\mathrm{T}}^{\mathrm{t}}$ of the $t \bar{t}$ system. Figure 4 (upper row) shows the differential results as a function of $\left|y_{t \bar{t}}\right|$ and $m_{\mathrm{tt}}$. The inclusive measurement using $19.7 \mathrm{fb}^{-1}$ of lepton+jets events yields an asymmetry of $A_{C}=0.0010 \pm 0.0068$ (stat) \pm 0.0037 (syst).

The same unfolding method is applied to $19.5 \mathrm{fb}^{-1}$ of dilepton data (electrons or muons) [15] to derive the $\Delta|y|$ distribution on parton level. One feature of the dilepton channel is that one can also analyze purely lepton based charge asymmetries, which do not rely on a full reconstruction of the $t \bar{t}$ system. The advantage of lepton based sensitive variables like the difference of the absolute values of the rapidities of the two leptons, $\Delta|\eta|$, is the absence of distorting effects from the $\bar{t} \bar{t}$ reconstruction, resulting in only small corrections trough the unfolding procedure which in turn leads to smaller uncertainties. In both cases, lepton asymmetry and charge asymmetry, inclusive measurements as well as differential measurements are performed. Figure 4 (lower row) shows the charges asymmetry (left) and the lepton asymmetry (right) as a function of the invariant mass of the $\mathrm{t} \overline{\mathrm{t}}$ system, using the same binning as in the lepton+jets analysis. The measured inclusive asymmetries are $A_{C}=0.011 \pm 0.011$ (stat) \pm 0.007 (syst) and $A_{C}^{\text {lep }}=0.003 \pm 0.006$ (stat) \pm 0.003 (syst). 

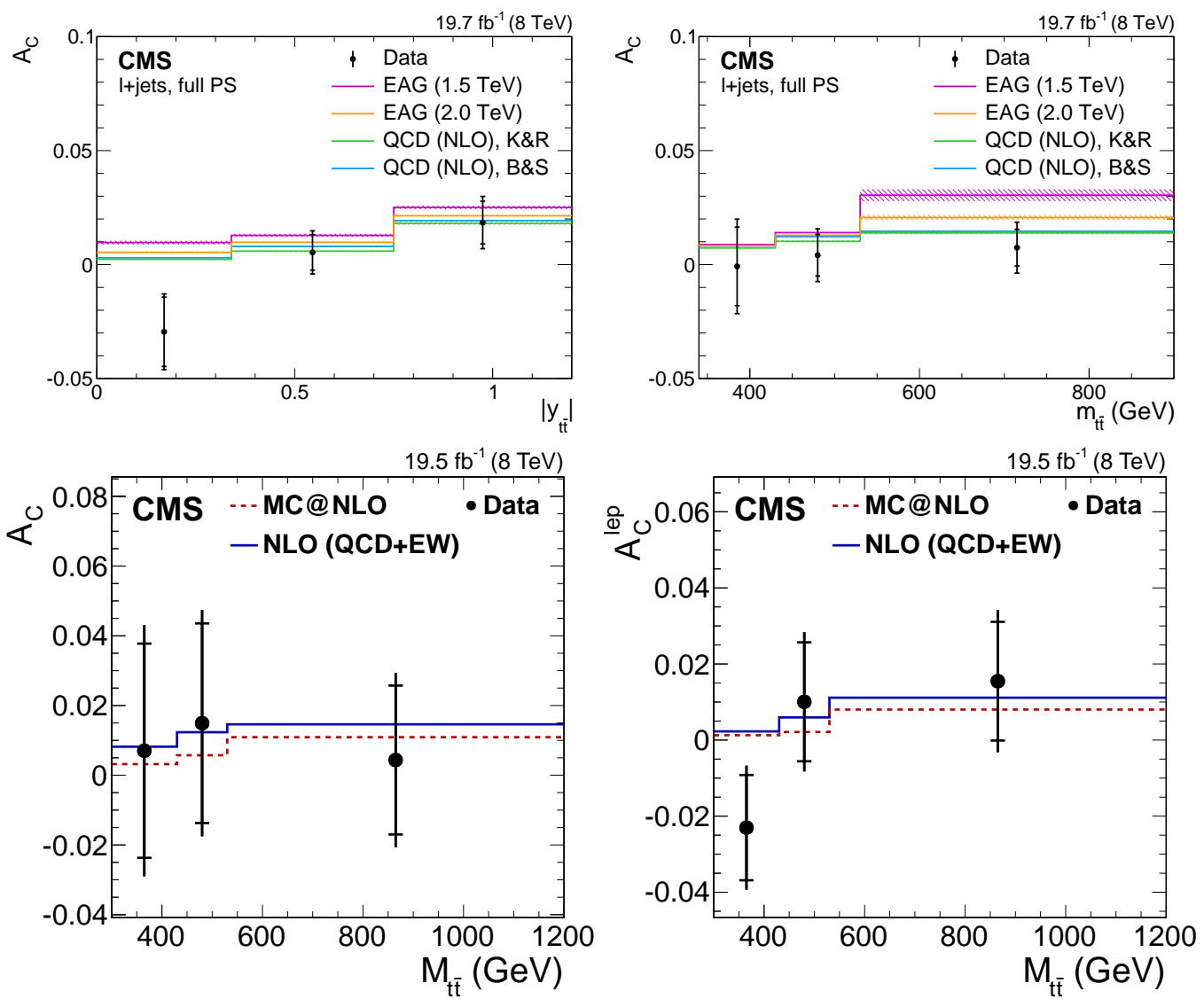

Figure 4: Charge asymmetry measurements using an unfolding method at CMS. Upper row: Lepton+jets [10]: Corrected asymmetry as a function of $\left|y_{\mathrm{tt}}\right|$ (left) and $m_{\mathrm{tt}}$ (right). The measured values are compared to NLO predictions for the SM based on calculations by Kühn and Rodrigo (K\&R) [7] and Bernreuther and $\mathrm{Si}(\mathrm{B} \& \mathrm{~S})[8,12]$, as well as to the predictions of a model featuring an effective axial-vector coupling of the gluon (EAG) [13, 14]. Bottom row: Dilepton [15]: Dependence of the asymmetries $A_{C}$ (left) and $A_{C}^{\text {lep }}$ (right), obtained from the unfolded distributions in data, on $M_{\mathrm{t}}$. The inner bars indicate the statistical uncertainties, while the outer bars represent the statistical and systematic uncertainties added in quadrature. The last bin of each plot includes overflow events.

\subsection{Measurement of the t⿱t charge asymmetry in lepton+jets and dilepton events using an unfolding method at ATLAS}

The ATLAS lepton+jets analysis [16] uses data corresponding to an integrated luminosity of $20.3 \mathrm{fb}^{-1}$. To measure the charge asymmetry, the full $\mathrm{t} \overline{\mathrm{t}}$ system is reconstructed using a kinematic fit that assesses the compatibility of the observed event with the tt pair kinematics using a likelihood approach. The reconstructed $\Delta|y|$ distribution is unfolded to parton level using the Fully Bayesian Unfolding (FBU) technique [17]. In addition to the inclusive measurement, ATLAS measures $A_{C}$ as a function of $m_{\mathrm{t} \mathrm{t}}, p_{\mathrm{T}}^{\mathrm{t} \overline{\mathrm{t}}}$, and the boost in $z$-direction, $\beta_{z, \mathrm{t}}$. The treatment of systematic uncertainties is consistently included by extending the likelihood entering the posterior probability density computation with nuisance parameter terms which are marginalized. The inclusive $\bar{t} \bar{t}$ charge asymmetry is measured to be $A_{C}=0.009 \pm 0.005$ (stat $\oplus$ syst). 

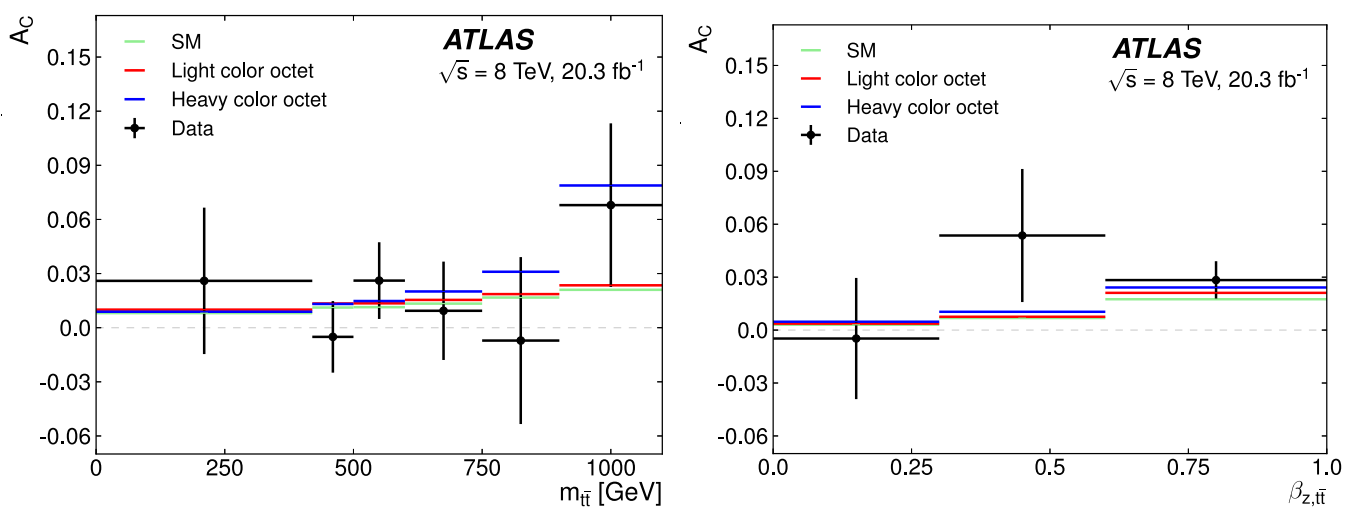

Figure 5: Charge asymmetry measurements using an unfolding method on ATLAS lepton+jets events [16]. Measured $A_{C}$ values as a function of bin-averaged $m_{\overline{\mathrm{t}}}$ and $\beta_{z, \mathrm{tt}}$, compared with predictions for SM [8] and for right-handed color octets with masses below the tt threshold and beyond the kinematic reach of current LHC searches [19].
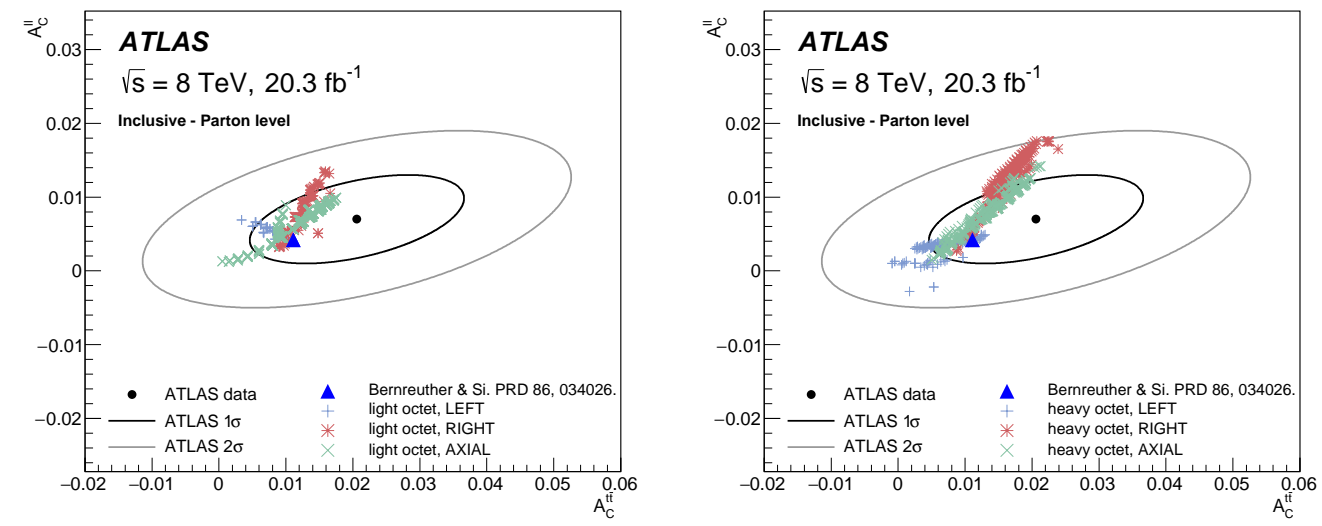

Figure 6: Charge asymmetry measurements using an unfolding method on ATLAS dilepton events[18]. Comparison of the inclusive $A_{C}^{\ell \ell}$ and $\mathrm{t} \bar{t}$ measurement values in the full phase space to the SM NLO QCD+EW prediction [8] and to two benchmark BSM models [19], one with a light octet with mass below the th production threshold (left) and one with a heavy octet with mass beyond the reach of the LHC (right), for various couplings as described in the legend. Ellipses corresponding to $1 \sigma$ and $2 \sigma$ combined statistical and systematic uncertainties of the measurement, including the correlation between $A_{C}^{\ell \ell}$ and $\mathrm{t} \overline{\mathrm{t}}$ are also shown.

With the same integrated luminosity ATLAS analyzes also dilepton events [18]. As in the CMS dilepton analysis the $t \bar{t}$ charge asymmetry is measured using $\Delta|y|$ of the top quarks and the lepton asymmetry is measured using $\Delta|\eta|$ of the two leptons. Asymmetries are measured inclusively as well as differentially as a function of $m_{\mathrm{t}}, p_{\mathrm{T}}^{\mathrm{t}}$, and $\beta_{z, \mathrm{t}}$. The reconstructed distributions are corrected to the parton level by applying the FBU method. The inclusive results are $A_{C}^{\ell \ell}=0.008 \pm$ 0.006 (stat $\oplus$ syst) for the lepton asymmetry and $A_{C}=0.021 \pm 0.016$ (stat $\oplus$ syst) for the charge asymmetry. Figure 6 shows these inclusive results in a 2-dimensional $A_{C}^{\ell \ell}-A_{C}$ plane together with predictions from two benchmark models for physics beyond the standard model (BSM). 
This analysis as well as the analysis in the lepton+jets channel by CMS [10] provides results not only for an extrapolation to the full phase space but also for a fiducial volume, based on detector acceptance and selection requirements. As the fiducial measurements do not rely on extrapolations to regions of the phase space that are not within the detector acceptance they are less dependent on a correct modelling of far-flung regions of the phase space for $t \bar{t}$ production. The measurements in the full phase space on the other side have the benefit of being directly comparable to theoretical calculations at the parton level, including predictions by exotic physics models, and we restrict ourselves in this document therefore to the results for the full phase space.

\subsection{Measurement of the t⿱t charge asymmetry in boosted events at ATLAS}

In a special version of the lepton+jets analysis discussed above the ATLAS Collaboration looks into events with a boosted topology, where the decay products of the hadronically decaying top quark are collinear and end up in one large jet [20]. This region is defined by the invariant mass of the t⿱t system being larger than $750 \mathrm{GeV}$ and the difference of the absolute values of the rapidities of top quark and antiquark, $\Delta|y|$ has to be between -2.0 and $+2.0 . \Delta|y|$ is also used as sensitive variable, and its unfolded distribution (using the FBU method) is used to obtain the charge asymmetry for all events with $m_{\mathrm{t}}>750 \mathrm{GeV}$ and differentially in three bins of $m_{\mathrm{tt}}$. Figure 7 (left) shows the inclusive and differential results. The measured inclusive asymmetry is $A_{C}=$ $0.042 \pm 0.032$ (stat $\oplus$ syst $)$.
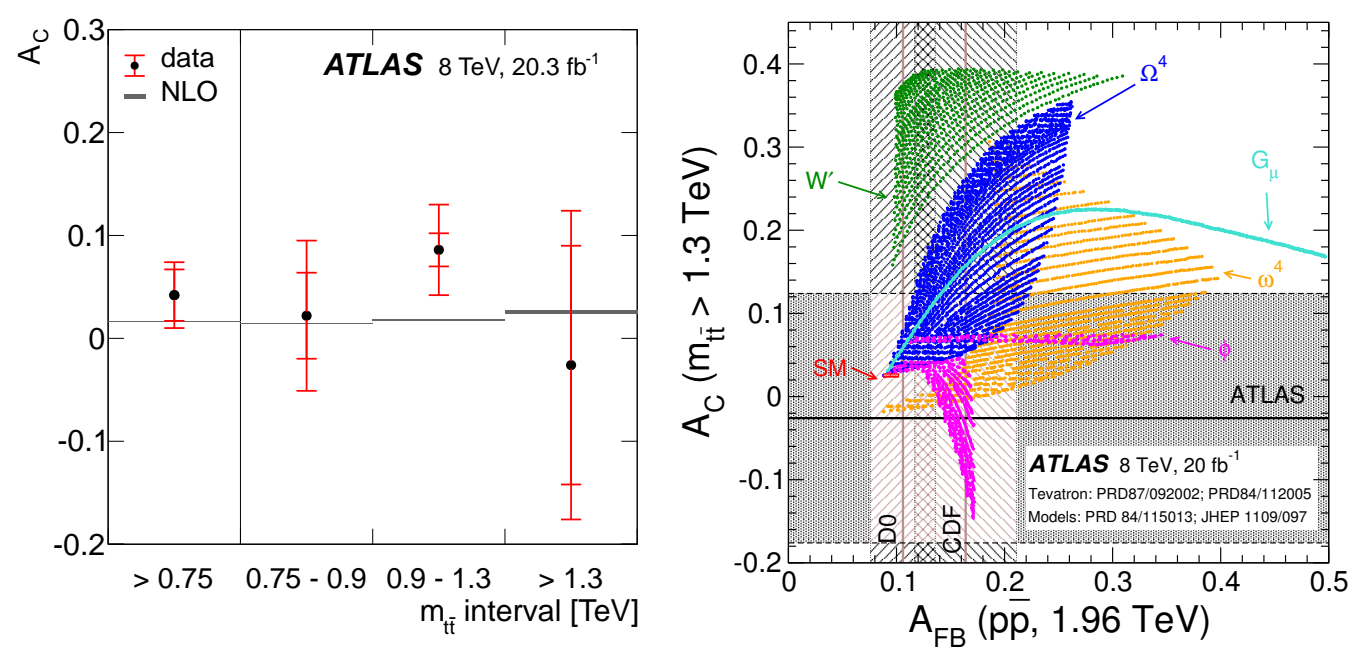

Figure 7: Charge asymmetry measurements in boosted events [20]. Left: $A_{C}$ measurements in different intervals of $m_{\mathrm{t} \mathfrak{t}}$. The error bars on the data indicate the modelling and unfolding systematic uncertainties (inner bar) and the total uncertainty, which includes the statistical uncertainty and the experimental systematic uncertainties. The SM prediction of the NLO calculation [7] for $A_{C}$ of top-quark pairs with $|\Delta y|<2$ is indicated as a shaded horizontal bar in each $m_{\mathrm{t}}$ bin, where the width of the bar indicates the uncertainty. Right: Measured inclusive charge asymmetries $A_{C}$ at the LHC versus forward-backward asymmetries $A_{F B}$ at Tevatron, compared with the SM predictions as well as predictions incorporating various potential BSM contributions: a $\mathrm{W}^{\prime}$ boson, a heavy axigluon $\left(G_{\mu}\right)$, a scalar isodoublet $(\phi)$, a color-triplet scalar $\left(\omega^{4}\right)$, and a color-sextet scalar $\left(\Omega^{4}\right)$. The horizontal $68 \%$ confidence level bands and central lines correspond to the ATLAS measurement, while the vertical ones correspond to the CDF and D0 measurements. 
Figure 7 (right) summarizes the results of measurements of the forward-backward asymmetry at the Tevatron and the t⿱t charge asymmetry at the LHC with their uncertainties. The measured results are compared with the SM prediction as well as with several BSM predictions (see the caption of Fig. 7 for the list of scenarios included). The region with high invariant masses of the $t \bar{t}$ system is a good place to exclude (or discover) contributions from BSM physics.

\section{Summary}
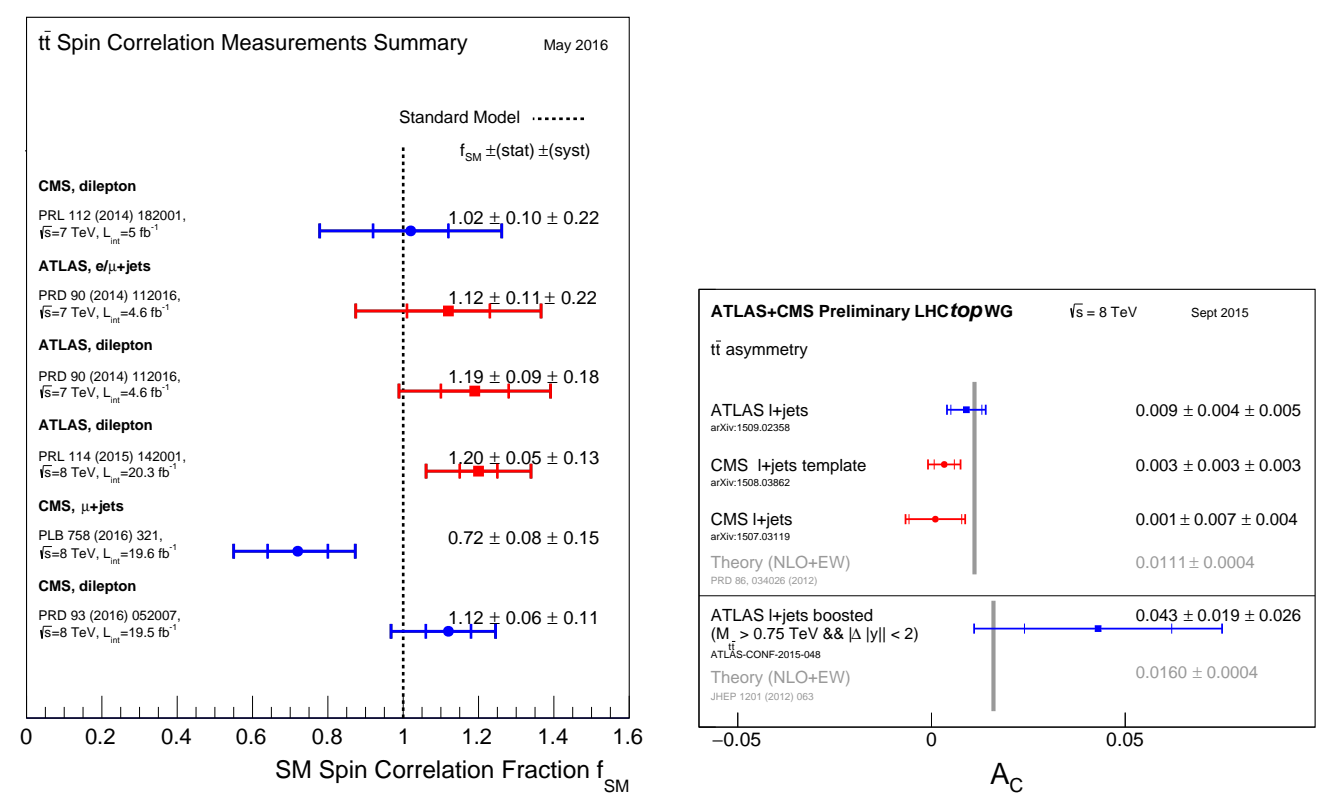

Figure 8: Left: Summary of measurements of SM t⿱t spin correlation fraction $f$ from ATLAS and CMS in different final states [21]. Right: Summary of the charge asymmetry measurements performed by ATLAS and CMS at $8 \mathrm{TeV}$, showing both the inclusive measurements and the measurement using boosted events with $m_{\mathrm{tt}}>0.75 \mathrm{TeV}$ and $|(\Delta(|y|))<2|[22]$.

Figure 8 summarizes the results of measurements of the spin correlation in $\bar{t} \bar{t}$ events and the results of measurements of the $t \bar{t}$ charge asymmetry in events with a lepton+jets final state. All results shown in this summary figure as well as the not-included asymmetry results from dileptonic $t \bar{t}$ events are comparable with the predictions by the standard model. No significant deviations that would point to new physics beyond the standard model could be found.

\section{References}

[1] ATLAS Collaboration, The ATLAS Experiment at the CERN Large Hadron Collider, JINST 3 S08003 (2008)

[2] CMS Collaboration, The CMS experiment at the CERN LHC, JINST 3 S08004 (2008)

[3] ATLAS Collaboration, Measurement of Spin Correlation in Top-Antitop Quark Events and Search for Top Squark Pair Production in pp Collisions at $\sqrt{s}=8 \mathrm{TeV}$ Using the ATLAS Detector, Phys. Rev.

Lett. 114142001 (2015) [hep-ex/1412.4742] 
[4] S. Frixione, B.R. Webber, Matching NLO QCD computations and parton shower simulations, JHEP 06029 (2006) [hep-ph/ 0204244 ]

[5] CMS Collaboration, Measurements of $\bar{t}$ spin correlations and top quark polarization using dilepton final states in pp collisions at $\sqrt{s}=8 \mathrm{TeV}$, Phys. Rev. D 93052007 (2016) [hep-ex/1601.01107]

[6] CMS Collaboration, Measurement of spin correlations in $t \bar{t}$ production using the matrix element method in the muon+jets final state in pp collisions at $\sqrt{s}=8 \mathrm{TeV}$, Phys. Lett. B 758321 (2016) [hep-ex/1511.06170]

[7] J. Kühn and G. Rodrigo, Charge asymmetries of top quarks at hadron colliders revisited, JHEP 01 063 (2012) [hep-ph/1109.6830]

[8] W. Bernreuther and Z.-G. Si, Top quark and leptonic charge asymmetries for the Tevatron and LHC, Phys. Rev. D 86034026 (2012) [hep-ph/1205. 6580]

[9] CMS Collaboration, Measurement of the charge asymmetry in top quark pair production in pp collisions at $\sqrt{s}=8 \mathrm{TeV}$ using a template method, Phys. Rev. D 93034014 (2016) [hep-ex/1508.03862]

[10] CMS Collaboration, Inclusive and differential measurements of the $t \bar{t}$ charge asymmetry in pp collisions at $\sqrt{s}=8 \mathrm{TeV}$ Phys. Lett. B 757154 (2016) [hep-ex/1507.03119]

[11] S. Schmitt, TUnfold, an algorithm for correcting migration effects in high energy physics, JINST 7 (2012) T10003 [physics.data-an/1205.6201]

[12] W. Bernreuther and Z.-G. Si, Distributions and correlations for top quark pair production and decay at the Tevatron and LHC, Nucl. Phys. B 83790 (2010) [hep-ph/1003. 3926]

[13] E. Gabrielli, M. Raidal, and A. Racioppi, Implications of the effective axial-vector coupling of gluon on top-quark charge asymmetry at the LHC, Phys. Rev. D 85074021 (2012) [hep-ph/1112 . 5885]

[14] E. Gabrielli and M. Raidal, Effective axial-vector coupling of gluon as an explanation to the top quark asymmetry, Phys. Rev. D 84 (2011) [hep-ph/1106.4553]

[15] CMS Collaboration, Measurements of $t \bar{t}$ charge asymmetry using dilepton final states in pp collisions at $\sqrt{s}=8 \mathrm{TeV}$, Phys. Lett. B $\mathbf{7 6 0} 365$ (2016) [hep-ex/1603.06221]

[16] ATLAS Collaboration, Measurement of the charge asymmetry in top-quark pair production in the lepton-plus-jets final state in pp collision data at $\sqrt{s}=8 \mathrm{TeV}$ with the ATLAS detector, Eur. Phys. J. C 7687 (2016) [hep-ex/1509.02358]

[17] G. Choudalakis, Fully Bayesian Unfolding, [hep-ph/1201.4612]

[18] ATLAS Collaboration, Measurements of the charge asymmetry in top-quark pair production in the dilepton final state at $\sqrt{s}=8 \mathrm{TeV}$ with the ATLAS detector (2016) [hep-ex/1604.05538]

[19] J.A. Aguilar-Saavedra, Portrait of a colour octet, JHEP 08172 (2014) [hep-ph/14 05.5826 ]

[20] ATLAS Collaboration, Measurement of the charge asymmetry in highly boosted top-quark pair production in $\sqrt{s}=8 \mathrm{TeV}$ pp collision data collected by the ATLAS experiment, Phys. Lett. B 75652 (2016), [hep-ex/1512.06092]

[21] https://twiki.cern.ch/twiki/bin/view/CMSPublic/PhysicsResultsTOPSummaryFigures

[22] https://twiki.cern.ch/twiki/bin/view/LHCPhysics/LHCTopWGSummaryPlots 